Europhysics Letters

PREPRINT

\title{
Heterogeneous Dynamics, Marginal Stability and Soft Modes in Hard Sphere Glasses
}

\author{
Carolina Brito ${ }^{1}$, Matthieu Wyart $^{2}$ \\ 1 Instituto de Física, Universidade Federal do Rio Grande do Sul, 91501-970 Porto \\ Alegre, Brazil \\ 2 Division of Engineering and Applied Sciences, Harvard University, Pierce Hall, 29 \\ Oxford Street, Cambridge, Massachusetts 02138, USA
}

PACS. 81.05.Rm - Porous material, granular material.

PACS. 82.70.-y -Disperse systems, Complex fluids.

PACS. 64.70.Pf - Glass transitions.

\begin{abstract}
. -
In a recent publication we established an analogy between the free energy of a hard sphere system and the energy of an elastic network [1. This result enables one to study the free energy landscape of hard spheres, in particular to define normal modes. In this Letter we use these tools to analyze the dynamics, both in the aging regime deep in the glass phase and near the glass transition. We observe numerically that structural relaxation occurs mostly along a small number of nearly-unstable, extended modes. This result, at odds with current spatial descriptions of the glass transition, furnishes a natural explanation for the collective aspect of the relaxation and for the length scales at play.
\end{abstract}

A colossal effort has been made to characterize the spatial nature of the structural relaxation near the glass transition. Numerical simulations 2 and experiments [3, 4, have shown that the dynamics in super-cooled liquids is heterogeneous. Both the string-like [5] and the compact [6] aspects of the particles' displacements have been emphasized. Nevertheless, the cause of such collective motions remains debated [7, 8. To make progress, one would like to relate these motions to other objects. A possible candidate is the excess of low-frequency modes present in all glasses, the so-called boson peak [9. Because these modes shift in general to lower frequencies as the temperature increases toward the glass transition temperature $T_{g}$, it has been proposed that they are responsible for the melting of the glass [10, 11. This suggests the use of widely employed tools, such as the low-frequency instantaneous normal modes [12] or the negative directions of saddles of the potential energy landscape [13, to analyze the collective motions causing relaxation. Nevertheless, this approach has the major drawback of being based on energy instead of free energy. As such, it cannot be applied for example to hard spheres or colloids, where structural relaxation is also known to be collective, see e.g. 44. In this case barriers between meta-stable states are purely entropic. More generally, one expects entropic effects to be important for glasses where hard-core repulsions and non-linearities are not negligible, which is presumably the case in general near $T_{g}[14$.

(c) EDP Sciences 
Recent developments make this analysis possible in hard sphere systems. In [1], we derived an analogy between the free energy of a hard sphere glass and the energy of a weakly-connected network of logarithmic springs. This allows us to define normal modes, that can be compared with the dynamics. Furthermore, recent results [15, 16, 17, valid for weakly-connected networks, such as elastic particles near jamming [18, 19] - where scaling laws between packing geometry and vibrational properties were first observed - or simple models of silica [20], apply to characterize these modes: (i) Excess modes appear above some frequency $\omega_{A M}$ which depends on the pressure $p$ and the coordination $z$, whose definition shall be recalled below for hard spheres. These anomalous modes extend at least on a length scale $l^{*}$, which depends on $z$ and diverges near maximum packing [16, 17, 19. (ii) Meta-stable states can exist only if they contain a configuration for which $\omega_{A M}>0$. This leads to a relationship between $p$ and $z$ that must be satisfied in the glass phase. Numerically, we observed that the hard sphere glass lies close to marginal stability: the coordination is just sufficient to maintain rigidity [1]. This implies that anomalous modes are present at very low-frequency.

In this Letter, we study how these modes take part in the structural relaxation, both during the aging dynamics deep in the glass phase, and in the vicinity of the glass transition where the system is at equilibrium. We show that when relaxation occurs, the system yields in the direction of the softest anomalous modes: most of the amplitude of the observed displacements can be decomposed on a small fraction of the modes, of the order of few percent. This observation supports the claim that the collective aspect of the relaxation does not stem from the non-linear coupling of localized relaxation events, as is supposed in most spatial models of the glass transition or in the common interpretation of the mode-coupling theory [22, but rather from the extended character of the softest degrees of freedom.

We start by recalling some results of [1. In a meta-stable state of a hard sphere system, one can define a contact network [23]: two particles are said to be in contact if they collide during some interval of time $t_{1}$, where $t_{1}$ is chosen to be much larger than an $\tau_{c}$, the collision time, and smaller than the structural relaxation time $\tau$ where meta-stability is lost. An approximation of the Gibbs free energy $\mathcal{G}$ can then be expressed as a sum on all the contacts $\langle i j\rangle$ :

$$
\mathcal{G}=-k T \sum_{\langle i j\rangle} \ln \left(\left\langle h_{i j}\right\rangle_{t}\right)
$$

where $h_{i j}=r_{i j}-r_{i}-r_{j}$ is the gap between particles $i$ and $j, r_{i j}$ is the distance between them, $r_{i}$ denotes the radius of particle $i$, and \langle\rangle$_{t}$ is a time-average. Eq. (11) has two main limitations: (i) it is only exact near the maximum packing fraction $\phi_{c}$ where the pressure diverges and (ii) to perform the time-average one requires a strong separation of time scales between $\tau_{c}$ and $\tau$. Thus Eq.(11) is a better approximation deep in the glass phase. Nevertheless the corrections to Eq.(1) are found to be rather small empirically [1, 17, and we shall use Eq. (11) to study the vicinity of the glass transition $\left(\phi \approx \phi_{0}\right)$ as well.

Eq.(1) can be expanded around any equilibrium position. For a contact $i j$, one finds for the force $V_{i j}^{\prime}=-k T /\left\langle h_{i j}\right\rangle_{t}$ and for the stiffness $V_{i j}^{\prime \prime}=k T /\left\langle h_{i j}\right\rangle_{t}^{2}$. This enables one to compute the dynamical matrix $\mathcal{M}$ [25] which relates a small applied force to the linear displacement of the average particle positions. Normal modes can then be computed, whose angular frequencies are the square roots of the eigenvalues of $\mathcal{M}$. In what follows we locate quiet periods of the dynamics where $\mathcal{M}$ can be estimated. Then, we use the normal modes to analyze the subsequent structural relaxation.

We consider a bidisperse two-dimensional hard sphere system. Half of the particles have a diameter $\sigma_{1}=1$, the other a diameter 1.4, their mass is $m=1$, and energies are expressed in units of $k T$. To study the aging dynamics, configurations are generated in the glass phase 

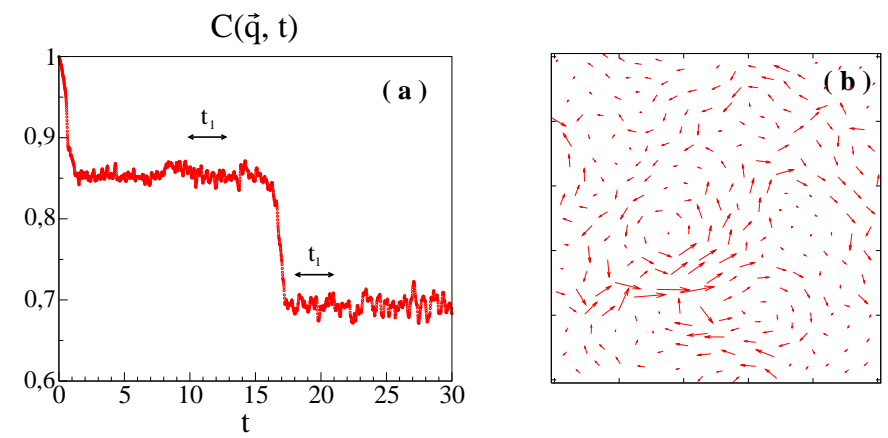

Fig. 1 - Left: Self-density correlation function $C(\vec{q}, t) v s$. time for $q=4 \pi / \sigma_{1}$ in a system of $N=256$ particles, at packing fraction $\phi=0.837$. Meta-stable states appear as plateaus of $C(\vec{q}, t)$, whereas the drops of $C(\vec{q}, t)$ are the mentioned earthquakes. Time-averages are made during the time segments $t_{1}$. Right: Displacement field of the corresponding earthquake. Arrows connect the average particles position before and after the earthquake, they are amplified 4 times here for visibility.

$\left(\phi_{0} \approx 0.79 \leq \phi \leq \phi_{c} \approx 0.84\right)$ as in [1]. An event-driven code is used to simulate the dynamics. We observe long quiet periods, or meta-stable states, interrupted by sudden rearrangements, or "earthquakes". Such earthquakes correspond to collective motions of a large number of particles. They have been observed in various other aging systems, such as colloidal paste, laponite or Lennard-Jones simulations 24. They appear as drops in the self scattering function $C(\vec{q}, t) \equiv\left\langle\exp \left[i \vec{q} \cdot\left(\vec{R}_{i}(t)-\vec{R}_{i}(0)\right)\right]\right\rangle_{i}$, where \langle\rangle$_{i}$ is an average on all particles and $\vec{R}_{i}(t)$ is the position of particle $i$ at time $t$. An example of earthquake is shown in Fig.(1).

In what follows the average particles position in a meta-stable state $l$ is denoted $\left|R^{l}\right\rangle \equiv$ $\left\{\left\langle\vec{R}_{i}\right\rangle_{t}\right\}, i=1 \ldots N$. In practice the time-average \langle\rangle$_{t}$ is done on a long time $t_{1}$ corresponding to few hundred collisions per particle (we use $t_{1}=10^{5}, 5 \times 10^{4}$ numerical time steps for respectively $N=1024$ and 256 particles). The earthquake displacement field $\left|\delta R^{e}\right\rangle$ between two meta-stable states $l$ and $m$ is then defined as $\left|\delta R^{e}\right\rangle \equiv\left|R^{m}\right\rangle-\left|R^{l}\right\rangle$, see Fig(1-b). During earthquakes, we find that the average particles displacement is typically $10 \%$ of the particle diameter.

To analyze these displacement fields, we compute the average of the particles' positions and the contact network in the meta-stable state prior to the earthquake $\left({ }^{1}\right)$. This enables us to define $\mathcal{M}$ and the normal modes $\left|\delta R^{\alpha}\right\rangle$, where the label $\alpha=1, \ldots, 2 N$ ranks the modes by increasing frequencies $\omega^{\alpha}$. An example of the density of states $D(\omega)$ is shown in Fig.(2-a), together with the lowest frequency mode, Fig.(2-b). We indeed observe extended anomalous modes at very low frequencies, in agreement with the marginal stability inferred from the microscopic structure of the glass [1. Note that we occasionally observe a few unstable modes even deep in the glass phase, implying the presence of saddles (and multiple configurations of free energy minima) or "shoulders" in the meta-stable states we are considering. In the present work we do not focus on this aspect, and treat unstable modes as the rest.

We then project the earthquake displacement $\left|\delta R^{e}\right\rangle$ on the modes and compute $c_{\alpha}=$ $\left\langle\delta R^{e} \mid \delta R^{\alpha}\right\rangle /\left\langle\delta R^{e} \mid \delta R^{e}\right\rangle$, where $\left\langle\delta R^{e} \mid \delta R^{\alpha}\right\rangle \equiv \sum_{i} \delta \overrightarrow{R_{i}^{e}} \cdot \delta \overrightarrow{R_{i}^{\alpha}}$. The $c_{\alpha}$ 's satisfy $\sum_{\alpha} c_{\alpha}{ }^{2}=1$ since the normal modes form a unitary basis. To study how the contribution of the modes depends on frequency, we define $g(\omega)=\left\langle c_{\alpha}{ }^{2}\right\rangle_{\omega}$, where the average is made on all $\alpha$ such that $\omega^{\alpha} \in$

$\left({ }^{1}\right)$ Very close to $\phi_{c}\left(\right.$ for $\left.\langle f\rangle>5 \times 10^{3}\right)$, "rattlers" [18] are present, which are systematically removed from our analysis [1. 


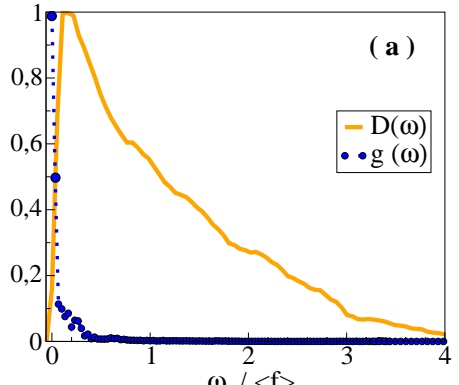

$\omega /<f>$

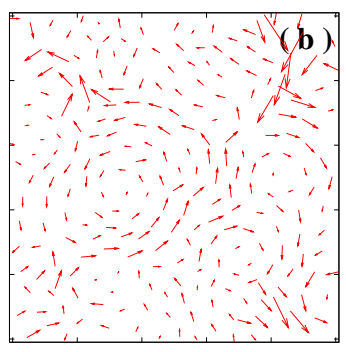

Fig. 2 - Left, straight curve: $D(\omega)$ computed in the meta-stable state prior to the earthquake of Fig 1 vs. $\omega /\langle f\rangle$, the angular frequency rescaled by the average contact force $\langle f\rangle$, computed by averaging on all pairs of particles in contact. Dotted curve: $g(\omega) v s . \omega /\langle f\rangle$. Right: Lowest frequency mode.

$[\omega, \omega+d \omega]$. Fig.(2-a) shows $g(\omega)$ for the earthquake shown in Fig.(11). We see that the average contribution of the modes decreases fast with increasing frequency. This implies that a small fraction of the modes contribute to the relaxation.

To make this observation systematic, we introduce the label $i$ to rank the $c$ 's by decreasing order: $c_{1}>c_{2} \ldots>c_{2 N}$. Then we define:

$$
F(k) \equiv \sum_{i=1}^{k} c_{i}^{2}
$$

$F(k)$ indicates which fraction of the total displacement is contained in the $k$ most contributing modes. If $F(k)=1 \forall k$ then only one mode contributes. If $F(k)=k / 2 N$ all modes contribute equally. We then define $k_{1 / 2}$ as the minimum number of modes contributing to $50 \%$ of the displacements, i.e. the smallest $k$ for which $F(k)>1 / 2$. Fig.(3) shows $F(k)$ and $F_{1 / 2} \equiv$ $k_{1 / 2} /(2 N)$ for the 17 cracks studied. We find $F_{1 / 2}<2 \%$ throughout the glass phase. We checked that this fraction is systematically smaller when only the beginning of the earthquake is considered [26].

We now extend this analysis to an equilibrated super-cooled liquid. We are interested here in how a region yields collectively, and not how such regions interact with each other. Because the size $l$ of these regions decreases toward the liquid phase 2, we consider a rather small system of 64 particles for which we expect $L<l$, where $L$ is the system size. We equilibrate it for $0.77 \leq \phi \leq 0.786$. As previously observed e.g. in [6], the dynamics is heterogeneous in time, and sudden rearrangements still occur on time scales of the order of $\tau$, the time scale of the $\alpha$-relaxation $\left({ }^{2}\right)$. These collective displacements span the entire system for $N=64$. We use the procedure previously described to determine the average configuration of meta-stable states, and to define the displacement relaxing the structure, see Fig.(4-a). We study five packing fractions. In each case, $F(k)$ and $F_{1 / 2}$ are computed for seven relaxation events. Results for $\left\langle F_{1 / 2}\right\rangle$ are presented in Fig. (44). To check the robustness of our procedure we compared two time-averaging windows: $t_{1}=10^{4}$ and $t_{1}=5 * 10^{3}$ numerical time steps. We find that $\left\langle F_{1 / 2}\right\rangle \leq 5 \%$ for all $\phi$ studied, implying that in this region of the super-cooled liquid phase, only a small fraction of the low-frequency modes contribute to the relaxation events. Interestingly, this fraction decays significantly as the relaxation time grows, suggesting a rarefaction of the number of directions along which the system can yield near

$\left({ }^{2}\right)$ We define $\tau$ as the time for which $C(\vec{q}, \tau)=0.3$. 

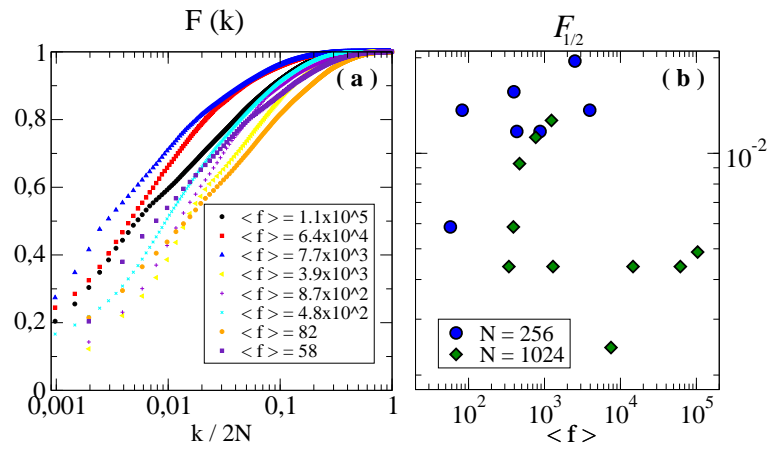

Fig. 3 - Left: Examples of $F(k) v s . k / 2 N$ for systems with different average contact force $\langle f\rangle$. $\langle f\rangle$ is measured before the earthquake. It is proportional to the pressure near $\phi_{c}$, and is of the order of 20 near the glass transition $\phi_{0}$. Right: $F_{1 / 2}$ vs. $\langle f\rangle$ for $N=256$ (diamond) and $N=1024$ (circle) particles.

the glass transition. We leave a detailed analysis of this question for future studies. Fig. (5) shows the comparison between a relaxation event and the mode that contributed most to it.

If the free or periodic boundaries of a system (or subsystem) of size $L$ are suddenly fixed, the lowest frequency of the anomalous modes inside increases as long as $L \leq l^{*}[15$. For this statement to be true, any linear combination of the lowest-frequency anomalous modes must have a spatial extension $l \geq l^{*}$. Thus the assumption that the structural relaxation occurs along the softest directions of the free energy, supported in the high-density range we probed
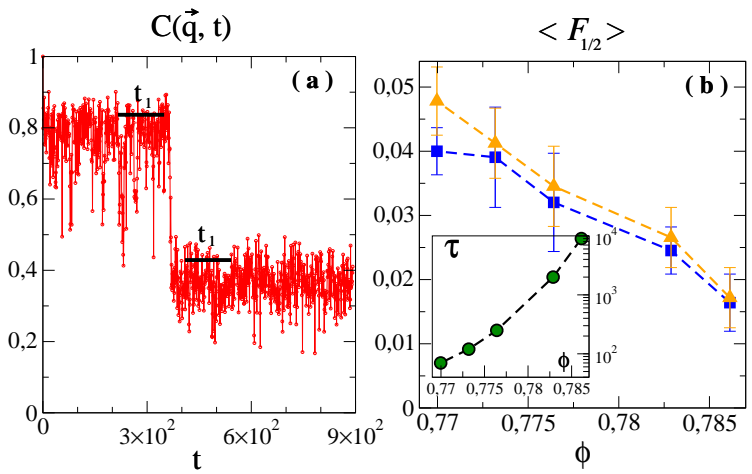

Fig. 4 - Left: $C(\vec{q}, t)$ in an equilibrated system of $N=64$ particles at $\phi=0.786$. The segment $t_{1}$ corresponds to $10^{4}$ numerical time steps. Right: $\left\langle F_{1 / 2}\right\rangle$ vs $\phi$ for $t_{1}=5 \times 10^{3}$ (triangle) and $t_{1}=10^{4}$ (square). Inset: relaxation time $\tau$ vs $\phi$. 
EUROPHYSICS LETTERS
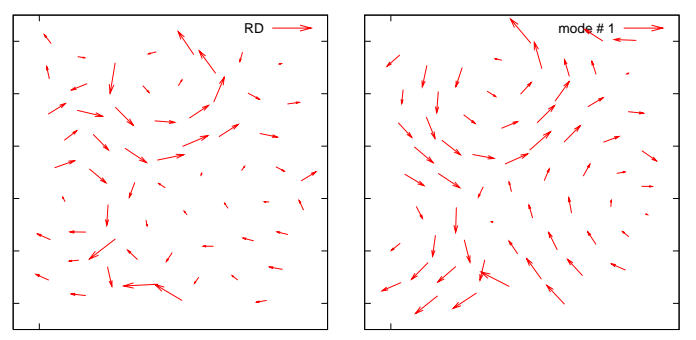

Fig. 5 - Left: Real displacement field corresponding to the relaxation of Fig.(4-a). Arrows were multiplied by 1.2. Right: lowest frequency normal mode, which has the highest projection in this particular case. In this example one mode contributes to most of the displacement.

by Fig.(3) and (4), leads to the conclusion that regions of size at least $l^{*}$ must rearrange. Deep in the glass phase our simulations certainly satisfy $l^{*} \geq L$ since $l^{*}$ diverges near maximum packing. We believe we are in the same situation in the small system $N=64$ at the glass transition. This is consistent with the observation that both the modes and the structural relaxation extend on the system size. From this analysis we expect that the dynamics slows down when boundary conditions are suddenly fixed at a distance $L \leq l^{*}$, the distance at which the frequency of the softest modes is affected.

In conclusion, we have introduced a method to analyze the free energy landscape of hard sphere glasses, and we have used it to study the intermittent dynamics occurring in the aging regime and in the vicinity of the glass transition. We have observed that the relaxation is dominated by the softest directions of the free energy. This result could be tested for other interaction potentials, such as Lennard-Jones systems or model of silica glass, which also present anomalous modes at low-frequency [21, 17. Note that all the theoretical framework used here applies identically in three dimensions, where we expect our results to be valid as well.

The hard sphere glass is marginally stable towards extended soft modes, and these modes dominate the structural relaxation. These observations are hard to reconcile with a description where the glass transition is induced by the freezing of local structures such as cages. Spatial models of the transition in which the relaxation is governed by local rules, such as models of dynamical facilitation, can generate collective structural relaxations. Nevertheless they cannot explain the observed linear marginal stability of the glass, nor why relaxation is dominated by a few extended modes. These models do not capture the fact that the relevant degrees of freedom are extended, rather than local. This is, we believe, the main cause for the widely observed collective aspect of the structural relaxation. This also raises the hope that a simple description of the dynamics in terms of a few (but collective) degrees of freedom will improve our understanding of the dynamical arrest characterizing the glass transition.

We thank J-P. Bouchaud, L. G. Brunnet, D. Fisher, O. Hallatschek, S. Nagel and T. Witten for helpful discussion and L.Silbert for furnishing the initial jammed configurations. C. Brito was supported by CNPq and M. Wyart by the Harvard Carrier Fellowship. 


\section{REFERENCES}

[1] C. Brito and M. Wyart, Europhys. Lett., 76 (1), pp. 149-155 (2006)

[2] see e.g. M.M. Hurley and P. Harrowell, Phys. Rev. E 52, 1694 (1995); Y. Hiwatari and T. Muranaka, J. Non-Cryst. Solids, 235-237, 19 (1998); A. Widmer-Cooper and P. Harrowell, Phys. Rev. Lett. 96, 185701 (2006)

[3] M. T. Cicerone and M. D. Ediger, J. Chem. Phys. 103, 5684 (1995); L. Berthier, G. Biroli, J-P. Bouchaud, L. Cipelletti, D. El Masri, D. L’Hote, F. Ladieu, M. Pierno; Science 310, 1797 (2005).

[4] E.R. Weeks, J.C. Crocker, A.C. Levitt, A. Schofield, D.A. Weitz, Science, 287 (5453): 627-631 (2000)

[5] C. Donati, J. F. Douglas, W. Kob, S. J. Plimpton, P. H. Poole, and S. C. Glotzer, Phys. Rev. Lett. 80, 2338 (1998); S.C. Glotzer, J. Non-Cryst. Solids, 274 (1-3): (2000)

[6] G. A. Appignanesi, J. A. Rodriguez Fris, R. A. Montani, and W. Kob, Phys. Rev. Lett. 96, 057801 (2006)

[7] C. Toninelli, M. Wyart, L. Berthier, G. Biroli, and J-P. Bouchaud, Phys. Rev. E 71, 041505 (2005)

[8] G. Tarjus, S.A. Kivelson, Z. Nussinov, and P. Viot, J. Phys.-Cond. Matter 17 (50): R1143-R1182 (2005)

[9] Amorphous Solids, Low Temperature Properties, edited by W. A. Phillips (Springer, Berlin, 1981).

[10] S.Alexander, Phys. Rep.,296, 65 (1998)

[11] G. Parisi, Eur. Phys. J.E., 9 213-218 (2002)

[12] T. Keyes, J. Phys. Chem. A 101, 2921 (1997).

[13] T.S. Grigera, A. Cavagna, I. Giardina, G.Parisi, Phys. Rev. Lett., 88, 055502 (2002); D. Coslovich and G. Pastore, Europhys. Lett., 75 (5), 784-790 (2006)

[14] D. Chandler, J.D. Weeks, H.C. Andersen, Science 220 (4599): 787-794 1983

[15] M. Wyart, S.R. Nagel, T.A. Witten, Europhys. Lett., 72, 486-492, (2005)

[16] M. Wyart, L.E.Silbert, S.R. Nagel, T.A. Witten, Phys. Rev. E 72051306 (2005)

[17] M. Wyart, Ann. Phys. (Paris), 30, No. 3 (2005) pp.1-96, or arXiv cond-mat/0512155

[18] C.S O'Hern, L.E Silbert, A. J. Liu and S.R. Nagel, Phys. Rev. E, 68, 011306 (2003)

[19] L. E. Silbert, A. J. Liu, and S. R. Nagel, Phys. Rev. Lett 95, 098301 (2005).

[20] K. Trachenko, M.T. Dove, V. Brazhkin and F.S. El'kin Phys. Rev. Lett. 93, 135502 (2004)

[21] N. Xu, M. Wyart, A. J. Liu, S. R. Nagel, arxiv cond-mat 0611474

[22] Gotze W. and Sjorgen L., Rep. Prog. Phys.,241 (1992)

[23] A. Ferguson, B. Fisher, B. Chakraborty, Europhys. Lett., 66, 277 (2004)

[24] W. Kob W, JL. Barrat, F. Sciortino,. P. Tartaglia J., Phys. Condensed Matter 126385 (2000); A.Duri, P Ballesta, L. Cipelletti, H. Bissig and V. Trappe, Fluctuation and Noise Lett.,5, 1-15, (2005); L Buisson, L Bellon and S Ciliberto, J. Phys.: Condens. Matter 15 S1163S1179 (2003)

[25] Neil Ashcroft and N.David Mermin, Solid state physics, New York (1976).

[26] C. Brito and M. Wyart, in preparation 